\title{
The Present Research Situation and Prospects about the Cement Sheath Defect Affecting on Connection Strength of Casing
}

\author{
Hua Tong ${ }^{1}$, Xiao-Hong Tang ${ }^{2}$ \\ Mechanical and Electrical Engineering, Southwest Petroleum University, Chengdu, Sichuan, China
}

\begin{abstract}
At present, in domestic and foreign cementing process, there is often a cementing quality without qualified. The cement sheath defects caused by too low displacement efficiency and gas channeling, will greatly reduce the casing bearing capacity, reduce the service life of the casing, so studying about cement sheath defects affecting on casing strength is very important. Through access to a large number of reference, summarizes the currently, at home and abroad, researches about cement sheath defects affecting on casing strength, analyzes the main technical difficulties faced by, and looks forward to the development trend of the research, point out that we should bind site cement sheath defects with actual situation, build a more close to the reality of cement sheath defect model, establish a set of more perfect cement sheath defects research system for influence on casing strength to further improve the cement sheath defect morphology. In order to improve the research level of the casing strength, the laboratory experiment and field investigation, computer tomography technology and computer aided software technology are studied in the paper.
\end{abstract}

Keyword cement sheath; defects; casing; the connection strength; security

\section{Introduction}

As for cement sheath, its function is to reduce and delay strata surrounding rock react on the casing for improving the force situation of the casing, and prolong its life. When the cementing quality is bad, the cement sheath defects occur. Casing will bear non-uniform extrusion load from formation, which reduces the bearing capacity of the casing, and affects the service life of casing.

Large domestic oilfield drilling data shows: In Daqing oil field, 1985-1986, successive arises four large casing damage areas. There were 528 wells' casings emerging damage during this time. At 1991-1995, found 1115 casing damage Wells, and until the end of 2007 the cumulative oil and gas well casing damage were found 10300 , accounts for the production is $18.17 \%$ of the total number of Wells. Since the first casing damage Wells was found by Shengli oilfield, the quantity and proportion of the casing damage are increasing year by year. A census in 1992 showed that a total of 1659 oil and gas Wells casing exist varying degrees of injury, accounting for about $10 \%$ of the total number of Wells, by 2003, the statistics of 27000 producing Wells shows that there were 5400 casing damage Wells, up to $20 \%$ of casing damage.

To sum up, the problem of casing damage has become a big problem to restrict oil industry, also shows the importance and necessity of the research of casing damage. So the researches about the cement sheath defects affecting on casing strength become very important.

\section{Geometry research of cement sheath defects}

Geometry research of cement sheath defects is based on studying about cement sheath defects affecting on casing strength. Due to the complex diversity of cement sheath defects, the main researches at home and abroad from the theoretical to simplify the cement sheath defects, thus establish the geometrical model of it. Geometric model currently used of the cement sheath defects, mostly is to assume that the defects of the cement sheath at a certain angle part missing completely.

In 2012, Zhu X H based on this model, combined with the cement sheath parameters such as defect shape, defect depth and number of defect. In 1995, the Cernocky E P discusses the cement sheath defect geometry model under the limiting cases of horizontal section in horizontal well. In 2012, Sugden $\mathrm{C}$ made an assumption that there is a dog leg at the well section, and what are the cement sheath defects like.

\section{Researches about cement sheath defects affecting on casing strength}

\subsection{The conventional finite element model}

In non-eccentric vertical wells, cement defects geometry model with complete absence of part of the cement is the most straightforward kind of geometry model, finite element calculation model is established based on the defect geometry model, which is currently the largest studied at home and abroad.

In 2007, Yang X W studied about cement sheath defects affecting on casing connection strength, and showed that cementing quality of cement sheath had great effects on the strength of the casing. Establish a finite element model of wellbore plane strain with cement sheath missing 1/12. Make the simulation analysis by cement sheath defects affecting on casing strength. Draw some conclusions that without internal pressure the maximum radial displacement of casing are 3.9 times when cement sheath intact, missing cement made Mises stress concentration factor 2.0, the outer well of casing maximum plastic strain reaches 0.0152 . Quantitative analysis of the cementing quality impact on the casing strength, provide quantitative data for the thermal recovery well casing damage. 2011, Cao C et al. established 


\section{International Journal of Science and Research (IJSR) \\ ISSN (Online): 2319-7064 \\ Index Copernicus Value (2013): 6.14 | Impact Factor (2015): 6.391}

the cement sheath model lack of $30^{\circ}, 60^{\circ}, 90^{\circ}$ Angle of the plane strain finite element model. Make the simulation analysis about cement sheath defects causing casing stress changes, then clear the degree on the relationship between the strength of casing and cement ring defects.

In 2012, Zou A Q et al. through the finite element simulation, made calculation and analysis about the cement sheath affecting on internal pressure strength of casing, which obtained some laws of amount of residual affecting on internal pressure strength. Meanwhile make the finite element calculation when cement sheath was under defects. Get the variation of the casing remainder internal pressure strength under the different defects angles. 2012, Lssa A. Kalil did the numerical simulation study of casing force condition in case on some cement and other complete deletion, obtained the size of the casing strength effect with the cement completely deletions.

From the above findings, after the cement sheath existing defects, first with increasing defect angle increased, Mise stress dramatically increase, then reduce again, there will be a slight increase again when the defect reaches about $270^{\circ}$, and at finally reduce the process, especially cement sheath defect reaches about $60^{\circ}$, the casing Mise stress reaches the maximum stress, serious concentration happened. Therefore, when the cement defects at some angle, due to stress concentration, not only cannot enhance the internal pressure capability of the casing, but reduce the compressive strength of the casing.

\subsection{Finite element defect model under certain conditions}

On the basis of the conventional model, research and analysis casing strength change in certain special formations, construction conditions or other special circumstances, which is also currently at home and abroad mainly research directions.

In 2002, Pattillo P D and Kristianse T G established a finite element calculation model with cement defects in horizontal well, simulation cement sheath stress changes along with time under different opening of defects to sheath. In the same year, Song M, used the computer-aided engineering (CAE) method to study the cement sheath defects, cement sheath not centered and the casing carrying capacity caused by nature of cement sheath. Propose the basic mechanisms that poor quality of cementing leading casing damage.

In 2004, Berger A, applied the Ansys software respectively simulating casing maximum stress under the condition of different pore pressure in soft formation, with defects opening from $20^{\circ}$ to $180^{\circ}$. Proves that under the same conditions with the increase of pore pressure, the force influence, that defects' opening degree has to casing, is gradually weakened; When the defects' opening degree is fixed, within a certain range, the higher the pore pressure is, the smaller casing stress is.

In 2007, Yang X W used ANSYS software to simulate the casing stress changes caused by cement sheath defects, and pointed out the relationship between casing maximum plastic strain of outer wall and temperature.
In 2008, Chen Y for the study about affecting of thermal recovery wells cement in the circumferential direction of local damage on the mechanical properties of the casing, used ABAQUS finite element software to build models with different degrees of cement missing sheath in the circumferential direction, studied casing stress, displacement law in cyclic steam stimulation process.

In 2009, Yang B Q used APDL as development of tools to establish the finite element model, systematically studied the law of that in the non-uniform ground stress field, the inner wall of the casing wear position and cement sheath defect position affecting on casing stress.

In 2009, Manoochehr Salehabadi studied the effect of cement sheath thermal performance and mechanical properties on casing maximum stress, at the time of the formation containing gas hydrate dissociation, in the case of different cement defects' opening degree.

In 2009, Zhao $\mathrm{P}$ used finite element numerical method, making a study about cement sheath defects affecting on casing strength, under uniform and non-uniform ground stress, in thermal recovery and non-thermal recovery. Obtain the variation of casing force with cement sheath defect angle changing.

In 2012, Feng $\mathbf{J}$ apply similar criteria to design a similar casing wear model, and made combination experiments between different quality cementing defects and different wear. Combine the experimental results and finite element analysis to study the internal pressure strength under different cementing defects and different wear.

\subsection{The improved finite element model}

On the basis of the conventional model, in 2012, Zhu X H, joined some parameters such as the cement sheath defects shape, defects depth and number of defects, and established the coupling system finite element model including casing, cement sheath defects and formation. Coupling the temperature field and stress field, taking the casing strength reducing into consideration, studied cement sheath defects shape, the defects opening degree, the depth of defects and the number of defects on casing safety. But in this model, the defect position only appeared on the first page.

In the same year, Jerome Schubert, based on the above model, studied about cement sheath defects affecting on casing strength under plastic cement and brittle cement, when cement completely defected apart, defects on the first interface, defects on the second interface, cement defected internal, existed gas channel and casing eccentric. Get the more realistic conclusion and understanding. The current geometry model of most special defect is still in the theoretical, and lack of experimental supporting and reality-based, so studies about cement sheath defects affecting on casing strength under special conditions are also less.

\section{The main faced technical difficulties}

Currently, at home and abroad, the researches about cement

\section{Volume 5 Issue 6, June 2016 www.ijsr.net}




\section{International Journal of Science and Research (IJSR) \\ ISSN (Online): 2319-7064}

Index Copernicus Value (2013): 6.14 | Impact Factor (2015): 6.391

sheath defects affecting on casing strength are mainly focused on theoretical researches, with less combining with the actual situation. The current technical difficulties which constrain the researches about cement sheath defects affecting on casing strength are mainly the following two aspects.

\subsection{Under special conditions well cement defect shape is difficult to obtain}

Due to the lack of special equipment, tools, it is difficult to obtain actual form at the site, in the presence of specific well conditions with dogleg and the enlarged diameter borehole and other segments. Also the lack of relevant laboratory testing, results the current model for cement sheath defects only under specific well conditions remains at the experience research stage under the guidance of the theory.

\subsection{Morphological description of the cement sheath defect is difficult}

From the cement sheath defects at site and laboratory experiments under non-eccentric vertical well field conditions, the cement sheath defects' shape are complex, mostly irregular geometry. To establish a finite element model, representative and the calculation results with little error compared to the real, is greater difficulties.

\section{The prospects about the cement sheath defect affecting on connection strength of casing}

1) Morphology of cement ring defect. Use the advanced technology or experimental methods to obtain cement sheath defects in the form of various types of actual well conditions, to carry out statistical analysis of cement sheath defect morphology.

2) Geometry researches of cement sheath defects. Combined with the actual form of cement sheath defects, establish a geometry model of the cement sheath defect, more accurate, closer to the actual situation.

3) Establish a more comprehensive research system about cement sheath defects affecting on casing strength. By the researches, including cement sheath defects morphology statistical analysis, cement sheath defects geometry, cement defects simulation analysis of casing strength, about cement sheath defects affecting on casing strength, establish a complete set of research system about cement sheath defects affecting on casing strength.

\section{Conclusion}

1) Through the development in recent years, according to the cement sheath defects geometry, basically form the technical road that establishing the finite element model, and simulating the law of defects affecting on casing strength.

2) With the in-depth development of technical, here will face up to the difficulties and challenges are that defects model shape are single, quite different from the actual situation, and lacking a complete set of research system about studying about cement sheath defects affecting on casing strength.

3) Make laboratory tests and site researches are the effective protection to solve the current problem.

4) Applying computer tomography technology and computer-aided software technology is to improve level of technical support about the cement sheath defects affecting on the casing strength.

\section{Reference}

[1] Zhu X H, Liu S H, Tong H. Study on effects of in high temperature thermal recovery wells, cement sheath defects affecting on casing strength $[\mathrm{J}]$. Hydrodynamics Research and Development, 2012, 27(4):478- 485.

[2] Cernocky E P, Scholibo F C. Approach to Casing Design for Service in Compacting Reservoirs [J].SPE 30522, 1995.

[3] Sugden C, Johnson J, Chambers M. Special Considerations in the Design Optimization of the Production Casing in High- rate, Multistage- Fractured Shale Wells [J]. SPE151470,2012.

[4] Yang X W, Ju Y F. Studied about cement sheath defects affecting on casing connection strength $[\mathrm{J}]$. Oil Field Equipment, 2007, 36(9):49-52.

[5] Cao C, Zhou X L, Shi L H. Researches about relationship between cementing cement defects and casing bearing capacity $[\mathrm{J}]$. Concrete and cement products, 2011,12 (12):32-34.

[6] Zhou A Q, Mou Z L, Liu G. Study about cement sheath affecting on internal pressure strength $[\mathrm{J}]$. Science Technology and Engineering, 2012, 34(12):9328 -9333.

[7] Pattillo P D,Kristiansen T G. Analysis of Horizontal Casing Integrity in the Valhall Field[J].SPE/ISRM $78204,2002$.

[8] Song M, Yang F X, Song S L. The law of load capacity of cement affecting on casing $[\mathrm{J}]$. Oil Drilling \& Production Technology, 2002,24(4):7 -10.

[9] Berger A, Fleckenstein W W, Eustes A W. Effect of Eccentricity, Voids, Cement Channels, and Pore Pressure Decline on Collapse Resistance of Casing[J].SPE 90045,2004.

[10] Chen Y, Lian Z H, Chen M. Lack of coupling to the thermal stress analysis of thermal recovery wells wellbore cement circumferential $[\mathrm{J}]$. Oil Drilling \& Production Technology, 2008,4(2):108 -111.

[11]Pang B Q, Yang S, Dou Y H. Casing Wear and cement sheath defect position on Casing Stress [J]. Petroleum machinery, 2009, 37(10):1-3.

[12] Manoochehr Salehaba, Hooman Haghighi. Finite Element Modeling of Casing in Gas-Hydrate - Bearing Sediments [J].SPE113819, 2009.

[13] Zhao P , Zhao Z B, Liu L H. Cement ring missing on casing damage analysis [J]. Oil and Gas Field Surface Engineering, 2009, 28(10):40 -42.

[14] Yuan Zhaoguang,Schubert J,Teodoriu C. HPHT Gas Well Cementing Complications and its Effect on Casing Collapse Resistance [J]. SPE 153986,2012. 


\section{International Journal of Science and Research (IJSR) \\ ISSN (Online): 2319-7064}

Index Copernicus Value (2013): 6.14 | Impact Factor (2015): 6.391

[15] Haut R C, Crook R J. Laboratory Investigation of Lightweight, Low Viscosity Cementing Spacer Fluids[J].Society of Petroleum Engineers of AIME.1982:1828 -1834.

[16] Griffith J E, Sabins F L. Investigation of Ultrasonic and Sonic Bond Tools for Detection of Gas Channels in Cements [J].SPE 24573, 1992.

[17] De Andrade J, Torsaeter M and Opedal N. Influence of Casing Centralization on Cement Sheath Integrity During Thermal Cycling[J] IADC/SPE 168012,2014.

Volume 5 Issue 6, June 2016 www.ijsr.net 